\title{
COMPUTER TECHNOLOGY A CRT blanking circuit for video terminals
}

\author{
KEITH P. SENTIS and MARK B. BAROWICZ \\ University of Michigan, Ann Arbor, Michigan 48106
}

\begin{abstract}
A simple and inexpensive modification for the ADDS 980 video terminal is described which permits the characters displayed on the CRT to be blanked and restored (under software control). This circuit is useful in applications, such as RT measurement, where precise definition of stimulus onset is important. The technique described is general enough to be applied to a variety of other video terminals.
\end{abstract}

In experiments where video terminals are used for the presentation of stimulus information to subjects, the ability to precisely control the subject's access to this information is very important. However, the inherently serial nature of typical video terminals poses timing problems in these situations. As the magnitude of a given stimulus increases, so does the interval between the appearance of the initial segment of the stimulus (e.g., the first few characters) and completion of the stimulus generation. Even at high baud rates, the transmission to a terminal of a 12-line prose passage can take a full $1 \mathrm{sec}$. Intervals of this length are often unacceptable in situations where the subject's processing time is of interest. Solution of this problem requires a method of restricting the subject's access to the terminal's video output during the interval in which the stimulus is being transmitted. Upon completion of stimulus transmission and generation, the subject's access to the video output could be restored, thus presenting the entire stimulus instantaneously (i.e., within one raster scan, or $16.7 \mathrm{msec}$ ). Reaction time (RT) timers could then be made coincident with the moment of stimulus access to this degree of precision. This report describes such a method that has been implemented on the Applied Digital Data Systems (ADDS) 980 video terminal.

The circuit shown in Figure 1 was used to modify both the Consul and the MRD versions of the ADDS 980 terminal and has the following effects. When the terminal receives the "keyboard-lock" control command sequence (ASCII characters, ESC, EM), any characters displayed on the screen are blanked. In addition, the cursor disappears. The keyboard is also locked unless this function has been disabled by the user. The terminal will remain in this mode until the "keyboard-unlock" sequence of ESC, BEL is received. At this point, the contents of the terminal's memory will again be displayed as usual. The cursor will appear along with

The authors wish to thank William Uttal, Cecil Murray, and Art Shane for their suggestions on this project.

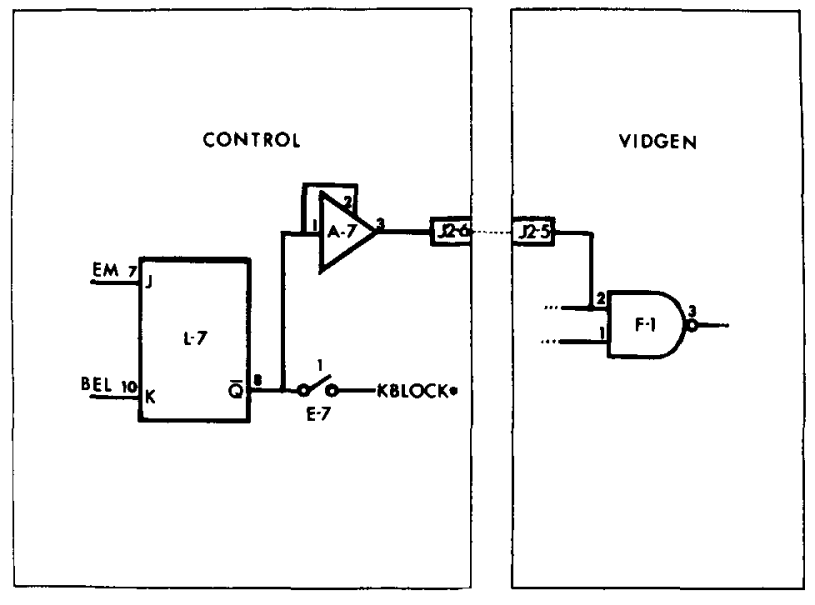

Figure 1. Modification circuit for ADDS 980 terminal to permit screen blanking under software control.

the characters displayed, and the keyboard will be unlocked.

This modification permits the following system of stimulus presentation. The screen is blanked by transmitting the "keyboard-lock" sequence. Next the stimulus material is transmitted in the usual manner with neither the characters nor the cursor appearing on the screen. Upon completion of the stimulus transmission, the keyboard-unlock sequence causes the stimulus to be displayed in its entirety. This procedure works identically with the graphics capability of the ADDS 980 terminal, allowing for the generation of fairly complex stimuli in different sectors of the screen without cuing the subjects as to which segment of the screen their attention will be directed to during the next trial.

As shown in Figure 1, the IC in Position L-7 on the Control PC board ${ }^{1}$ receives the decoded keyboard-lock command sequence ESC, EM and generates the KBLOCK* signal (which can be disabled with the mini-dip switch E7-1). The KBLOCK* signal (from Pin 8 of L-7) is used as input to a SN74125 Tri-State buffer (A-7) that has been added to the Control board as 
part of this modification. Normally, KBLOCK* is high and causes A-7 to present a high-impedance state. When KBLOCK* is asserted, A-7 presents a low impedance. The output of A-7 is transferred to the Vidgen PC board and is connected in parallel with Pin 2 of F-1, an input to the video mixing section. The input gated by $F-1$ is the serial video information containing the cursor image and any characters in the terminal's memory. Under normal conditions, the high impedance presented by A-7 has no effect on this input to the video mixer. However, receipt of the keyboard-lock command sequence causes A-7 to shunt the serial video signal at F-1 before it reaches the video mixing section, resulting in a blank screen.

Implementation of the modification is straightforward. Position A-7 on the Control card is empty and the SN74125 is installed there. Jumpers are installed on the cards as indicated in Figure 1. Transfer of the output of A.7 from the Control card to the Vidgen card can be accomplished by means of a jumper between the two cards. More conveniently, the connector tab J2-6 on the Control card can be jumped to tab J2-5 on the Vidgen card using the wire-wrap bay, thus allowing all wiring to be done on-board. On the Control card, tab J2-6 is an unused output (CLK*), and the foil should be severed between the connector J2-6 and the IC that produces CLK ${ }^{*}$. On the Vidgen card, the connector tab J2-5 is unused.

This technique is generally applicable to other models of terminals as well. All that is required is the installation of a Tri-State device that is activated and deactivated by particular signals. On many terminals, the screen-erase signal could be used to blank the screen, and the signal that sounds the audible alarm (BEL) could be used to restore it. The output of the Tri-State buffer could then be connected in parallel with an input to the video mixing section in a manner similar to the one described above.

\section{NOTE}

1. Complete schematics are available in How to Maintain the Consul 980. Applied Digital Data Systems, Inc., 100 Marcus Blvd., Hauppauge, New York 11787.

(Received for publication June 19, 1978; revision accepted July 18,1978 .) 